\title{
Some Novel Features in 2D Non-Abelian Theory: BRST Approach
}

\author{
N. Srinivas ${ }^{(a)}$, S. Kumar ${ }^{(a)}$, B. K. Kureel ${ }^{(a)}$ and R. P. Malik ${ }^{(a, b)}$ \\ (a) Physics Department, Centre of Advanced Studies, \\ Banaras Hindu University, Varanasi - 221 005, (U.P.), India \\ (b) DST Centre for Interdisciplinary Mathematical Sciences, \\ Institute of Science, Banaras Hindu University, Varanasi - 221 005, India \\ e-mails: seenunamani@gmail.com, sunil.bhu93@gmail.com, bkishore.bhu@gmail.com, \\ rpmalik1995@gmail.com
}

\begin{abstract}
Within the framework of Becchi-Rouet-Stora-Tyutin (BRST) formalism, we discuss some novel features of a two $(1+1)$-dimensional (2D) non-Abelian 1-form gauge theory (without any interaction with matter fields). Besides the usual off-shell nilpotent and absolutely anticommutating (anti-)BRST symmetry transformations, we discuss the off-shell nilpotent and absolutely anticommutating (anti-)co-BRST symmetry transformations. Particularly, we lay emphasis on the existence of the coupled (but equivalent) Lagrangian densities of the 2D non-Abelian theory in view of the presence of (anti-)coBRST symmetry transformations where we pin-point some novel features associated with the Curci-Ferrari (CF) type restrictions. We demonstrate that these CF-type restrictions can be incorporated into the (anti-)co-BRST invariant Lagrangian densities through the fermionic Lagrange multipliers which carry specific ghost numbers. The modified versions of the Lagrangian densities (where we get rid of the new CF-type restrictions) respect some precise symmetries as well as a couple of symmetries with CF-type constraints. These observations are completely novel as far as the BRST formalism, with proper (anti-)co-BRST symmetries, is concerned.
\end{abstract}

PACS numbers: 11.30.Pb, 03.65.-w, 11.30.-j.

Keywords: 2D non-Abelian theory; nilpotent (anti-)BRST symmetries; nilpotent (anti-) co-BRST symmetries; Curci-Ferrari type restrictions; fermionic Lagrange multipliers 


\section{Introduction}

In modern language, the gauge theories are characterized by the first-class constraints (in the terminology of Dirac's prescription for classification scheme of constraints [1, 2]) and their gauge symmetries are generated by these constraints. For the covariant canonical quantization of theories, based on the above gauge symmetries, one of the conceptually elegant, geometrically rich and theoretically beautiful methods is the Becchi-Rouet-StoraTyutin (BRST) formalism where a classical local gauge symmetry is traded with a couple of quantum gauge symmetries which are christened as the BRST and anti-BRST symmetries. The latter symmetries (i.e. BRST and anti-BRST) are, however, fermionic in nature as they are found to be nilpotent of order two. Hence, they are supersymmetric type, too.

We have established, in our recent publications, that a $p$-form $(p=1,2,3, \ldots)$ Abelian gauge theory in $D=2 p$ dimensions of spacetime respects, in addition to the (anti-)BRST symmetry transformations, the nilpotent and absolutely anticommuting (anti-)co-BRST symmetries, too (see, e.g. [3] and references therein). We have been able to demonstrate the existence of the latter type of symmetries in the 2D (non)-Abelian 1-form gauge theories, too, which has enabled us to establish that the 2D (non-)Abelian gauge theories (without any interaction with matter fields) provide a field theoretic example of Hodge theory [4,5] as well as a new model [6] of the topological field theory (TFT) which captures a few aspects of Witten-type of TFTs and some salient features of Schwarz-type TFTs.

In the above context, it is pertinent to point out that the above basic (anti-) BRST and (anti-)co-BRST symmetries are physically important because they provide physical realizations [6] of the cohomological operators of differential geometry and they establish the fact that the Lagrangian densities of 2D non-Abelian theory (cf. Eqn. (2) below) look like Witten-type TFT but the innate symmetry transformations of this theory resemble to that of the symmetry transformations of Schwarz-type TFTs (see, e.g. $[3,6]$ ). To be more specific, there is no shift symmetry in our theory which is one of the theoretical hallmark of a Witten-type TFT [7]. Rather, the symmetries of our present theory are internal in nature [8]. The topological invariants and their recursion relations of our present 2D theory have been obtained in our earlier work [6].

As far as discussion on TFTs of the 2D non-Abelain theory is concerned, it has been demonstrated (see. e.g. [6, 3]) that the Lagrangian density as well as symmetric energymomentum tensor of the theory can be expressed as the sum of the anticommutators with BRST and co-BRST charges. This observation is one of the key features of the Witten type TFT [7]. However, the symmetries of this theory do not include the shift symmetry as they are only internal in nature. Thus, the symmetries of this theory capture one of the decisive features of the Schwarz-type TFT [8]. These observations establish the novelty in the topological nature of our present theory. Thus, we note that the existence of the (anti-)BRST and (anti-)co-BRST symmetries is physically important and interesting.

The purpose of our present investigation is to point out a few novel features that are associated with the (anti-)co-BRST symmetry transformations in the context of 2D nonAbelian gauge theory (without any interaction with matter fields). We have focused on the coupled (but equivalent) Lagrangian densities $[9,10]$ in the Curci-Ferrari gauge [11, 12] which respect the proper (anti-)BRST symmetries on the constrained hypersurface (in the 2D spacetime manifold) where the CF-condition [13] is satisfied (cf. Eqn. (4) 
below). We have shown that the proof of the equivalence of the above coupled Lagrangian densities requires another set of CF-type restrictions w.r.t. the (anti-)co-BRST symmetry transformations. We have incorporated these latter CF-type restrictions in the modified versions of the Lagrangian densities (cf. Eqn. (8) below) which respect the (anti-)coBRST symmetry transformations separately and independently. We have commented on the origin of these new CF-type restrictions in view of the existing nilpotent (anti-)BRST and (anti-)co-BRST symmetry transformations of our present 2D non-Abelian theory.

Against the backdrop of the above statements, the central result of our present investigation is the proof of equivalence of the coupled Lagrangian densities (2) w.r.t. the (anti-)co-BRST symmetry transformations which requires new type of CF-type restrictions $(\mathcal{B} \times C)=0$ and $(\mathcal{B} \times \bar{C})=0$. The non-trivial solutions of these CF-type constraints are (i) the directions of the $\mathcal{B}$ and $C$ fields are parallel in the $\mathrm{SU}(\mathrm{N})$ Lie algebraic space for the antico-BRST invariance, and (ii) for the requirement of the co-BRST invariance, we observe that the direction of the field $\mathcal{B}$ in the $\mathrm{SU}(\mathrm{N})$ Lie algebraic space must be parallel to the direction of field $\bar{C}$. These are not very strong restrictions as co-BRST and anti-co-BRST symmetries are linearly independent of each-other because of their absolute anticommutativity property. In otherwords, the CF-type restrictions $\mathcal{B} \times C=0$ and $\mathcal{B} \times \bar{C}=0$ do not imply that $C$ and $\bar{C}$ fields are parallel to each-other in the Lie-algebraic space.

We call the restrictions $(\mathcal{B} \times C)=0$ and $(\mathcal{B} \times \bar{C})=0$ as CF-type restrictions from the point of view of symmetries (cf. Eqns. (4) and (7)). However, these restrictions can not be treated on equal footing to the original $\mathrm{CF}$ - condition $B+\bar{B}+(C \times \bar{C})=0$ because the latter emerge due to the $\mathrm{CF}$-gauge-fixing in the theory. To get rid of these new restrictions (i.e. $(\mathcal{B} \times C=0, \mathcal{B} \times \bar{C}=0)$, we have incorporated them into the Lagrangian densities (8) through the Lagrange multiplier fields in such a way that both the Lagrangian densities (8) respect the (anti-)co-BRST symmetry transformations separately and independently (cf. Eqns. (10) and (11) below). This is a novel observation in the sense that we can not perform such kind of exercise with the usual $\mathrm{CF}$-condition $B+\bar{B}+(C \times \bar{C})=0$ which is associated with the (anti-)BRST transformations of our present 2D non-Abelian theory.

The following key motivating factors have been at the heart of our present investigation. First, it is very important for us to establish the equivalence of the coupled Lagrangian densities (cf. Eqn. (2) below) w.r.t. the (anti-)co-BRST symmetry transformations because these symmetries play a very important role in the proof of the $2 \mathrm{D}$ non-Abelian theory to be a tractable field theoretic example of the Hodge theory as well as a new model of TFT. Second, the existence of the CF-type restrictions is one of the key signatures of a quantum gauge theory when the latter is discussed within the framework of BRST formalism. We have accomplished this goal in our present endeavor for the (anti-)BRST as well as (anti-)co-BRST symmetries. Finally, we have found the modified versions of the coupled Lagrangian densities (cf. Eqn. (8) below) which respect the (anti-)co-BRST symmetries separately and independently. All the above cited results are novel as far as the basic concepts behind the existence of (anti-)BRST and (anti-)co-BRST symmetry transformations for the Lagrangian densities of our 2D non-Abelian theory are concerned.

The contents of our present paper are organized as follows. In Sec. 2, we briefly recapitulate the bare essentials of the (anti-)BRST symmetry transformations for the 2D non-Abelian theory so that the convention and notations could be fixed. Our Sec. 3 is devoted to the discussion of (anti-)co-BRST symmetry transformations and existence of 
the CF-type restrictions. We comment on all the existing continuous symmetries of our theory in their operator form and obtain their algebra in Sec. 4. Finally, we make some concluding remarks in Sec. 5 .

\section{Preliminaries: (anti-)BRST symmetries}

We begin with the following 2D (anti-)BRST invariant coupled (but equivalent) Lagrangian densities $[9,10]$ in the Curci-Ferrari gauge (see, e.g. [11,12] for details)

$$
\begin{aligned}
\mathcal{L}_{B}^{(0)} & =-\frac{1}{4} F_{\mu \nu} \cdot F^{\mu \nu}+B \cdot\left(\partial_{\mu} A^{\mu}\right) \\
& +\frac{1}{2}(B \cdot B+\bar{B} \cdot \bar{B})-i \partial_{\mu} \bar{C} \cdot D^{\mu} C, \\
\mathcal{L}_{\bar{B}}^{(0)} & =-\frac{1}{4} F_{\mu \nu} \cdot F^{\mu \nu}-\bar{B} \cdot\left(\partial_{\mu} A^{\mu}\right) \\
& +\frac{1}{2}(B \cdot B+\bar{B} \cdot \bar{B})-i D_{\mu} \bar{C} \cdot \partial^{\mu} C,
\end{aligned}
$$

where the field strength tensor $F_{\mu \nu}=\partial_{\mu} A_{\nu}-\partial_{\nu} A_{\mu}+i\left(A_{\mu} \times A_{\nu}\right)$ has been defined through the 2-form $F^{(2)}=d A^{(1)}+i A^{(1)} \times A^{(1)}$ (with $d=d x^{\mu} \partial_{\mu}$ and $d^{2}=0$ ). The 1-form $A^{(1)}=d x^{\mu}\left(A_{\mu} \cdot T\right)$ defines the vector potential $A_{\mu}^{a}$ for the $2 \mathrm{D}$ non-Abelian theory ${ }^{*}$ and the Nakanishi-Lautrup type auxiliary fields $B$ and $\bar{B}$ obey the CF-condition $B+\bar{B}+(C \times \bar{C})=0$ where the fermionic $\left(C^{a} C^{b}+C^{b} C^{a}=0, \bar{C}^{a} \bar{C}^{b}+\bar{C}^{b} \bar{C}^{a}=0, C^{a} \bar{C}^{b}+\bar{C}^{b} C^{a}=0,\left(C^{a}\right)^{2}=\right.$ $\left(\bar{C}^{a}\right)^{2}=0$, etc.) (anti-)ghost fields $\left(\bar{C}^{a}\right) C^{a}$ are required for the validity of unitarity in our theory. The covariant derivative $D_{\mu} C=\partial_{\mu} C+i\left(A_{\mu} \times C\right)$ is in the adjoint representation of the $\mathrm{SU}(\mathrm{N})$ Lie algebra of our $2 \mathrm{D}$ non-Abelian theory.

For the 2D theory, it is clear that the kinetic term $\left[-\frac{1}{4}\left(F_{\mu \nu} \cdot F^{\mu \nu}\right)\right]$ is equal to: $-\frac{1}{2} F_{01}^{a} F^{01 a} \equiv \frac{1}{2} E^{a} E^{a}$ where $E^{a}=F_{01}^{a}=\partial_{0} A_{1}^{a}-\partial_{1} A_{0}^{a}+i\left(A_{0} \times A_{1}\right)^{a}$. This kinetic term can be linearized through the additional auxiliary field $\mathcal{B}$. The ensuing coupled Lagrangian densities are $[6,14]$ :

$$
\begin{aligned}
\mathcal{L}_{B} & =\mathcal{B} \cdot E-\frac{1}{2} \mathcal{B} \cdot \mathcal{B}+B \cdot\left(\partial_{\mu} A^{\mu}\right) \\
& +\frac{1}{2}(B \cdot B+\bar{B} \cdot \bar{B})-i \partial_{\mu} \bar{C} \cdot D^{\mu} C \\
\mathcal{L}_{\bar{B}} & =\mathcal{B} \cdot E-\frac{1}{2} \mathcal{B} \cdot \mathcal{B}-\bar{B} \cdot\left(\partial_{\mu} A^{\mu}\right) \\
& +\frac{1}{2}(B \cdot B+\bar{B} \cdot \bar{B})-i D_{\mu} \bar{C} \cdot \partial^{\mu} C .
\end{aligned}
$$

${ }^{*}$ We have taken into account the background 2D Minkowski spacetime manifold that is endowed with a flat metric with signatures $(+1,-1)$ and the Levi-civita tensor $\varepsilon_{\mu \nu}$ is chosen to be $\varepsilon_{01}=+1=\varepsilon^{10}$ and $\varepsilon_{\mu \nu} \varepsilon^{\nu \lambda}=\delta_{\mu}^{\lambda}$, etc., where the Greek indices $\mu, \nu, \lambda, \ldots=0,1$. In the $\mathrm{SU}(\mathrm{N})$ Lie algebraic space, we define the dot and cross products between two non-null vectors $P^{a}$ and $Q^{a}$ as $P \cdot Q=P^{a} Q^{a}$ and $(P \times Q)=f^{a b c} P^{b} Q^{c} T^{a}$ where $a, b, c, \ldots=1,2,3, \ldots N^{2}-1$. The generators $T^{a}$ satisfy the $\mathrm{SU}(\mathrm{N})$ Lie algebra $\left[T^{a}, T^{b}\right]=f^{a b c} T^{c}$ where the structure constants $f^{a b c}$ are chosen to be totally antisymmetric for the semi-simple Lie group SU(N). Throughout the whole body of our text, we denote the nilpotent (anti-) BRST and (anti-)co-BRST symmetry transformations by the notations $s_{(a) b}$ and $s_{(a) d}$, respectively. 
The above Lagrangian densities respect the following (anti-)BRST symmetry transformations $\left(s_{(a) b}\right)$, namely;

$$
\begin{aligned}
& s_{a b} A_{\mu}=D_{\mu} \bar{C}, s_{a b} \bar{C}=-\frac{i}{2}(\bar{C} \times \bar{C}), s_{a b} C=i \bar{B}, \\
& s_{a b} B=i(B \times \bar{C}), \quad s_{a b} E=i(E \times \bar{C}), \quad s_{a b} \bar{B}=0, \\
& s_{a b} \mathcal{B}=i(\mathcal{B} \times \bar{C}), s_{a b}\left(\partial_{\mu} A^{\mu}\right)=\partial_{\mu} D^{\mu} \bar{C}, s_{b} \bar{C}=i B \\
& s_{b} A_{\mu}=D_{\mu} C, s_{b} C=-\frac{i}{2}(C \times C), \quad s_{b} \bar{B}=i(\bar{B} \times C), \\
& s_{b} E=i(E \times C), \quad s_{b} \mathcal{B}=i(\mathcal{B} \times C), \quad s_{b} B=0,
\end{aligned}
$$

because the Lagrangian densities $\mathcal{L}_{B}$ and $\mathcal{L}_{\bar{B}}$ transform under $s_{(a) b}$ as (see, e.g. [14] for details):

$$
\begin{aligned}
s_{b} \mathcal{L}_{B} & =\partial_{\mu}\left[B \cdot D^{\mu} C\right], \quad s_{a b} \mathcal{L}_{\bar{B}}=\partial_{\mu}\left[-\bar{B} \cdot D^{\mu} \bar{C}\right] \\
s_{a b} \mathcal{L}_{B} & =\partial_{\mu}\left[-\{\bar{B}+(C \times \bar{C})\} \cdot \partial^{\mu} \bar{C}\right] \\
& +[B+\bar{B}+(C \times \bar{C})] \cdot D_{\mu}\left(\partial^{\mu} \bar{C}\right) \\
s_{b} \mathcal{L}_{\bar{B}} & =\partial_{\mu}\left[\{B+(C \times \bar{C})\} \cdot \partial^{\mu} C\right] \\
& -[B+\bar{B}+(C \times \bar{C})] \cdot D_{\mu}\left(\partial^{\mu} C\right) .
\end{aligned}
$$

Thus, we note that both the Lagrangian densities are equivalent in the sense that both of them respect the (anti-)BRST symmetry transformations on a hypersurface in the 2D spacetime manifold where the $\mathrm{CF}$-condition $B+\bar{B}+(C \times \bar{C})=0$ is satisfied. In other words, we have $s_{b} \mathcal{L}_{\bar{B}}=-\partial_{\mu}\left[\bar{B} \cdot \partial^{\mu} C\right]$ and $s_{a b} \mathcal{L}_{B}=\partial_{\mu}\left[B \cdot \partial^{\mu} \bar{C}\right]$.

We end this section with the following remarks. First, the (anti-)BRST symmetry transformations $s_{(a) b}$ are off-shell nilpotent $\left(s_{(a) b}^{2}=0\right)$ of order two which shows their fermionic (supersymmetric) nature. Second, the absolute anticommutating property $\left(s_{b} s_{a b}+s_{a b} s_{b}=0\right)$ is satisfied by the (anti-)BRST symmetry transformations $s_{(a) b}$ provided the CF-condition $B+\bar{B}+(C \times \bar{C})=0$ is invoked [13]. In other words, $s_{b}$ and $s_{a b}$ have their own identities on the hypersurface that is defined by the constraint equation $B+\bar{B}+(C \times \bar{C})=0$ and, therefore, they are linearly independent of each-other. Third, the coupled Lagrangian densities $\mathcal{L}_{B}$ and $\mathcal{L}_{\bar{B}}$ are equivalent only on the above hypersurface as far as the off-shell nilpotent and absolutely anticommuting (anti-)BRST symmetry transformations $s_{(a) b}$ are concerned. Finally, we observe that the total kinetic term [i.e. $-\frac{1}{4}\left(F_{\mu \nu} \cdot F^{\mu \nu}\right) \equiv \mathcal{B} \cdot E-\frac{1}{2} \mathcal{B} \cdot \mathcal{B}$, owing its origin to the exterior derivative (i.e. $d=d x^{\mu} \partial_{\mu}$ and $d^{2}=0$ ), remains invariant under the (anti-) BRST symmetry transformations of our present 2D theory. To be more specific, we have $F^{(2)}=\frac{1}{2}\left(d x^{\mu} \wedge d x^{\nu}\right)\left(F_{\mu \nu} \cdot T\right)=d A^{(1)}+i A^{(1)} \wedge A^{(1)}$ where $A^{(1)}=d x^{\mu}\left(A_{\mu} \cdot T\right)$ and $F_{\mu \nu}=\partial_{\mu} A_{\nu}-\partial_{\nu} A_{\mu}+i\left(A_{\mu} \times A_{\nu}\right)$. We observe that field strength tensor $F_{\mu \nu}$ owes its origin to the geometrical object $d=d x^{\mu} \partial_{\mu}$. 


\section{Nilpotent (anti-)co-BRST symmetry transforma- tions: Lagrangian formulation}

It is very interesting to note that the Lagrangian densities $\mathcal{L}_{B}$ and $\mathcal{L}_{\bar{B}}$ also respect another set of off-shell nilpotent $\left(s_{(a) d}^{2}=0\right)$ and absolutely anticommuting $\left(s_{d} s_{a d}+s_{a d} s_{d}=0\right)$ (anti-)co-BRST symmetry transformations $s_{(a) d}$, namely;

$$
\begin{aligned}
& s_{a d} A_{\mu}=-\varepsilon_{\mu \nu} \partial^{\nu} C, \quad s_{a d} C=0, \quad s_{a d} \bar{C}=i \mathcal{B}, \\
& s_{a d} \mathcal{B}=0, \quad s_{a d} E=D_{\mu} \partial^{\mu} C, \quad s_{a d} B=0, \\
& s_{a d} \bar{B}=0, \quad s_{a d}\left(\partial_{\mu} A^{\mu}\right)=0, \quad s_{d} A_{\mu}=-\varepsilon_{\mu \nu} \partial^{\nu} \bar{C}, \\
& s_{d} \bar{C}=0, \quad s_{d} C=-i \mathcal{B}, \quad s_{d} \mathcal{B}=0, \quad s_{d} B=0, \\
& s_{d} E=D_{\mu} \partial^{\mu} \bar{C}, \quad s_{d} \bar{B}=0, \quad s_{d}\left(\partial_{\mu} A^{\mu}\right)=0,
\end{aligned}
$$

because we observe that the Lagrangian densities transform to the following total spacetime derivatives, namely;

$$
s_{d} \mathcal{L}_{B}=\partial_{\mu}\left[\mathcal{B} \cdot \partial^{\mu} \bar{C}\right], \quad s_{a d} \mathcal{L}_{\bar{B}}=\partial_{\mu}\left[\mathcal{B} \cdot \partial^{\mu} C\right]
$$

which imply that the action integrals $\left(S=\int d^{2} x \mathcal{L}_{B}\right.$ and $\left.S=\int d^{2} x \mathcal{L}_{\bar{B}}\right)$ remain invariant under $s_{d}$ and $s_{a d}$ provided these transformations act, separately and independently, on $\mathcal{L}_{B}$ and $\mathcal{L}_{\bar{B}}$, respectively. In the above proof of symmetry invariance, we have used $\varepsilon_{\mu \nu}\left(\partial^{\mu} \bar{C} \times\right.$ $\left.\partial^{\nu} \bar{C}\right)=0$ as well as $\varepsilon_{\mu \nu}\left(\partial^{\mu} C \times \partial^{\nu} C\right)=0$ which are also useful in the proof of the absolute anticommuting and nilpotency properties of $s_{(a) d}$.

To establish the equivalenc $\dagger^{\dagger}$ of the Lagrangian densities $\mathcal{L}_{B}$ and $\mathcal{L}_{\bar{B}}$ w.r.t. (anti-)coBRST symmetry transformations, we observe that the following transformations are true:

$$
\begin{aligned}
s_{d} \mathcal{L}_{\bar{B}} & =\partial_{\mu}\left[\mathcal{B} \cdot D^{\mu} \bar{C}-\varepsilon^{\mu \nu}\left(\partial_{\nu} \bar{C} \times \bar{C}\right) \cdot C\right] \\
& +i \partial_{\mu} A^{\mu} \cdot(\mathcal{B} \times \bar{C}), \\
s_{a d} \mathcal{L}_{B} & =\partial_{\mu}\left[\mathcal{B} \cdot D^{\mu} C+\varepsilon^{\mu \nu} \bar{C} \cdot\left(\partial_{\nu} C \times C\right)\right] \\
& +i \partial_{\mu} A^{\mu} \cdot(\mathcal{B} \times C) .
\end{aligned}
$$

Thus, we note that both the Lagrangian densities $\mathcal{L}_{B}$ and $\mathcal{L}_{\bar{B}}$ can be equivalent w.r.t. the (anti-)co-BRST symmetry transformations, iff, we invoke the CF-type of restrictions as $\mathcal{B} \times \bar{C}=0, \mathcal{B} \times C=0$ which are physically allowed because both the above restrictions are perfectly (anti-)co-BRST invariant. In other words, we note that $s_{(a) d}[\mathcal{B} \times \bar{C}]=0, s_{(a) d}[\mathcal{B} \times$ $C]=0$. We call these restrictions as the CF-type restrictions by taking the analogy from Eqn. (4) where we have the CF-condition $(B+\bar{B}+C \times \bar{C}=0)$ in the context of nilpotent (anti-)BRST symmetry transformations. The above nomenclature has been adopted due to symmetry considerations only.

As far as the perfect (anti-)co-BRST symmetry transformations are concerned, the CFtype restrictions $(\mathcal{B} \times \bar{C}=0, \mathcal{B} \times C=0)$ can be incorporated into the modified versions of

\footnotetext{
${ }^{\dagger}$ By equivalence, we mean exactly similar kind of transformations as we have obtained in equation (4) for the (anti-)BRST symmetries which demonstrates that $\mathcal{L}_{B}$ and $\mathcal{L}_{\bar{B}}$ respect both these symmetries on the hypersurface where $B+\bar{B}+(C \times \bar{C})=0$.
} 
the Lagrangian densities $\mathcal{L}_{B}$ and $\mathcal{L}_{\bar{B}}$ as

$$
\begin{aligned}
& \mathcal{L}_{B} \rightarrow \mathcal{L}_{(B, \bar{\lambda})}=\mathcal{B} \cdot E-\frac{1}{2} \mathcal{B} \cdot \mathcal{B}+B \cdot\left(\partial_{\mu} A^{\mu}\right) \\
& +\frac{1}{2}(B \cdot B+\bar{B} \cdot \bar{B})-i \partial_{\mu} \bar{C} \cdot D^{\mu} C-\bar{\lambda} \cdot(\mathcal{B} \times C), \\
& \mathcal{L}_{\bar{B}} \rightarrow \mathcal{L}_{(\bar{B}, \lambda)}=\mathcal{B} \cdot E-\frac{1}{2} \mathcal{B} \cdot \mathcal{B}-\bar{B} \cdot\left(\partial_{\mu} A^{\mu}\right) \\
& +\frac{1}{2}(B \cdot B+\bar{B} \cdot \bar{B})-i D_{\mu} \bar{C} \cdot \partial^{\mu} C-\lambda \cdot(\mathcal{B} \times \bar{C}),
\end{aligned}
$$

where $\lambda$ and $\bar{\lambda}$ are the fermionic (i.e. $\lambda^{2}=0, \bar{\lambda}^{2}=0, \quad \lambda \bar{\lambda}+\bar{\lambda} \lambda=0$ ) Lagrange multiplier fields which carry the ghost number $(+1)$ and $(-1)$, respectively.

It is interesting to note that the above modified version of the Lagrangian density $\mathcal{L}_{(\bar{B}, \lambda)}$ respects the following perfect (anti-)co-BRST transformations $\left(s_{(a) d}\right)$ :

$$
\begin{aligned}
& s_{a d} A_{\mu}=-\varepsilon_{\mu \nu} \partial^{\nu} C, s_{a d} C=0, s_{a d} \bar{C}=i \mathcal{B}, \\
& s_{a d}(\mathcal{B} \times \bar{C})=0, s_{a d} E=D_{\mu} \partial^{\mu} C, s_{a d} \lambda=0, \\
& s_{a d}\left[\mathcal{B}, B, \bar{B},\left(\partial_{\mu} A^{\mu}\right)\right]=0, s_{d} C=-i \mathcal{B}, \\
& s_{d} A_{\mu}=-\varepsilon_{\mu \nu} \partial^{\nu} \bar{C}, s_{d}(\mathcal{B} \times \bar{C})=0, \quad s_{d} E=D_{\mu} \partial^{\mu} \bar{C}, \\
& s_{d} \lambda=i\left(\partial_{\mu} A^{\mu}\right), \quad s_{d} \bar{C}=0, \quad s_{d}\left[\mathcal{B}, B, \bar{B},\left(\partial_{\mu} A^{\mu}\right)\right]=0 .
\end{aligned}
$$

To corroborate on the above statement, we note that the Lagrangian density $\mathcal{L}_{(\bar{B}, \lambda)}$ transforms as follows:

$$
\begin{aligned}
& s_{a d} \mathcal{L}_{(\bar{B}, \lambda)}=\partial_{\mu}\left[\mathcal{B} \cdot \partial^{\mu} C\right] \\
& s_{d} \mathcal{L}_{(\bar{B}, \lambda)}=\partial_{\mu}\left[\mathcal{B} \cdot D^{\mu} \bar{C}-\varepsilon^{\mu \nu}\left(\partial_{\nu} \bar{C} \times \bar{C}\right) \cdot C\right] .
\end{aligned}
$$

The above observations establish that the action integral $\left(S=\int d^{2} x \mathcal{L}_{(\bar{B}, \lambda)}\right)$ remains invariant under $s_{(a) d}$. In exactly similar fashion, we observe that the Lagrangian density $\mathcal{L}_{(B, \bar{\lambda})}$ transforms as

$$
\begin{aligned}
& s_{d} \mathcal{L}_{(B, \bar{\lambda})}=\partial_{\mu}\left[\mathcal{B} \cdot \partial^{\mu} \bar{C}\right], \\
& s_{a d} \mathcal{L}_{(B, \bar{\lambda})}=\partial_{\mu}\left[\mathcal{B} \cdot D^{\mu} C+\varepsilon^{\mu \nu} \bar{C} \cdot\left(\partial_{\nu} C \times C\right],\right.
\end{aligned}
$$

under the following fermionic (anti-)co-BRST symmetry transformations $\left(s_{(a) d}\right)$

$$
\begin{aligned}
& s_{a d} A_{\mu}=-\varepsilon_{\mu \nu} \partial^{\nu} C, \quad s_{a d} C=0, \quad s_{a d} \bar{C}=i \mathcal{B}, \\
& s_{a d}(\mathcal{B} \times C)=0, s_{a d} E=D_{\mu} \partial^{\mu} C, \quad s_{a d} \bar{\lambda}=i\left(\partial_{\mu} A^{\mu}\right), \\
& s_{a d}\left[\mathcal{B}, B, \bar{B},\left(\partial_{\mu} A^{\mu}\right)\right]=0, s_{d} A_{\mu}=-\varepsilon_{\mu \nu} \partial^{\nu} \bar{C}, \quad s_{d} \bar{C}=0, \\
& s_{d} C=-i \mathcal{B}, s_{d}(\mathcal{B} \times C)=0, \quad s_{d} E=D_{\mu} \partial^{\mu} \bar{C}, \\
& s_{d} \bar{\lambda}=0, \quad s_{d}\left[\mathcal{B}, B, \bar{B},\left(\partial_{\mu} A^{\mu}\right)\right]=0,
\end{aligned}
$$

which are nilpotent of order two (i.e. $\left.s_{(a) d}^{2}=0\right)$ and absolutely anticommuting $\left(s_{d} s_{a d}+\right.$ $\left.s_{a d} s_{d}=0\right)$ in nature. As a side remark, we would like to emphasize that it is the gaugefixing term (owing its origin to the co-exterior derivative of differential geometry $\delta A^{(1)}=$ 
$\left.-* d * A^{(1)}=\partial_{\mu} A^{\mu}\right)$ that remains invariant under $s_{(a) d}$. To be more specific, the operator $\delta=-* d *$ is the co-exterior derivative where $*$ is the Hodge duality operation on the 2D flat Minkowski manifold.

At this juncture, we make the following remarks. First, we observe that the CF-type restrictions $(\mathcal{B} \times C=0, \mathcal{B} \times \bar{C}=0)$, in the context of (anti-)co-BRST symmetry transformations, are perfectly invariant under $s_{(a) d}$. Second, the CF-condition $(B+\bar{B}+C \times \bar{C}=0)$, in the context of (anti)BRST transformations $\left(s_{(a) b}\right)$, transforms under the (anti-)BRST symmetry transformations as:

$$
\begin{aligned}
& s_{b}[B+\bar{B}+(C \times \bar{C})]=i(B+\bar{B}+C \times \bar{C}) \times C, \\
& s_{a b}[B+\bar{B}+(C \times \bar{C})]=i(B+\bar{B}+C \times \bar{C}) \times \bar{C} .
\end{aligned}
$$

The above observations demonstrate that the celebrated CF-condition is (anti-) BRST invariant (cf. Eqn. (13)) only on the constrained hypersurface in the 2D spacetime manifold where the constrained field equation $(B+\bar{B}+C \times \bar{C}=0)$ is valid. Finally, the CF-type restrictions $(\mathcal{B} \times C=0, \mathcal{B} \times \bar{C}=0)$ could be incorporated in the Lagrangian densities through Lagrange multipliers $\lambda$ and $\bar{\lambda}$ (cf. Eqn. (8)) but the CF-condition $(B+\bar{B}+$ $C \times \bar{C}=0$ ) can not be incorporated in the Lagrangian densities so that one could have perfect symmetry invariance in the theory. In addition to the (anti-)co-BRST symmetry transformations $(9)$, the Lagrangian density $\mathcal{L}_{(\bar{B}, \lambda)}$ also respects the following anti-BRST symmetry transformations:

$$
\begin{aligned}
& s_{a b} A_{\mu}=D_{\mu} \bar{C}, \quad s_{a b} \bar{C}=-\frac{i}{2}(\bar{C} \times \bar{C}), \\
& s_{a b} C=i \bar{B}, \quad s_{a b} B=i(B \times \bar{C}), \quad s_{a b} \mathcal{B}=i(\mathcal{B} \times \bar{C}), \\
& s_{a b} \lambda=0, \quad s_{a b} E=i(E \times \bar{C}), \quad s_{a b} \bar{B}=0 .
\end{aligned}
$$

In exactly similar fashion, the Lagrangian density $\mathcal{L}_{(B, \bar{\lambda})}$ respects, besides the (anti-)coBRST symmetries (12), the following BRST symmetry transformations:

$$
\begin{aligned}
& s_{b} A_{\mu}=D_{\mu} C, s_{b} C=-\frac{i}{2}(C \times C), s_{b} \bar{C}=i B, \\
& s_{b} \bar{\lambda}=0, \quad s_{b} \bar{B}=i(\bar{B} \times C), \quad s_{b} E=i(E \times C), \\
& s_{b} \mathcal{B}=i(\mathcal{B} \times C), \quad s_{b} B=0 .
\end{aligned}
$$

In fact, we observe that $s_{a b} \mathcal{L}_{(\bar{B}, \lambda)}=\partial_{\mu}\left[-\bar{B} \cdot D^{\mu} \bar{C}\right]$ and $s_{b} \mathcal{L}_{(B, \bar{\lambda})}=\partial_{\mu}\left[B \cdot D^{\mu} C\right]$. As a consequence, we note that $\mathcal{L}_{(\bar{B}, \lambda)}$ and $\mathcal{L}_{(B, \bar{\lambda})}$ respect three perfect ${ }^{\ddagger}$ continuous fermionic symmetries at this stage.

It can be seen that, under the following nilpotent BRST symmetry transformations

$$
\begin{aligned}
& s_{b} A_{\mu}=D_{\mu} C, s_{b} C=-\frac{i}{2}(C \times C), s_{b} \bar{C}=i B, \\
& s_{b} \bar{B}=i(\bar{B} \times C), s_{b} E=i(E \times C), s_{b} \mathcal{B}=i(\mathcal{B} \times C), \\
& s_{b} B=0, \quad s_{b}\left(\partial_{\mu} A^{\mu}\right)=\partial_{\mu} D^{\mu} C, s_{b} \lambda=-i(\lambda \times C),
\end{aligned}
$$

\footnotetext{
${ }^{\ddagger}$ We do not invoke any CF-type restriction for the proof of the symmetry invariance in the theory Thus, the symmetries are perfect in the real sense of the word.
} 
the Lagrangian density $\mathcal{L}_{(\bar{B}, \lambda)}$ transforms as

$$
\begin{aligned}
s_{b} \mathcal{L}_{(\bar{B}, \lambda)} & =\partial_{\mu}\left[\{B+(C \times \bar{C})\} \cdot \partial^{\mu} C\right] \\
& -[B+\bar{B}+(C \times \bar{C})] \cdot D_{\mu}\left(\partial^{\mu} C\right) \\
& -i(\mathcal{B} \times \lambda) \cdot[B+C \times \bar{C}],
\end{aligned}
$$

which demonstrate that if we use the CF-conditions $B+\bar{B}+C \times \bar{C}=0$ as well as $\mathcal{B} \times \bar{B}=0$, we obtain the BRST invariance as $s_{b} \mathcal{L}_{(\bar{B}, \lambda)}=\partial_{\mu}\left[-\bar{B} \cdot \partial^{\mu} C\right]$ because the second term in (17) is zero due to the CF-condition $(B+\bar{B}+C \times \bar{C}=0)$ and the third term can be expressed as $i(\mathcal{B} \times \lambda) \cdot \bar{B} \equiv-i \lambda \cdot(\mathcal{B} \times \bar{B})$ which amounts to a $\mathrm{CF}$-type restriction $\mathcal{B} \times \bar{B}=0$. This restriction, however, is also equivalent to the restrictions $(\bar{B} \times \lambda)=0$ and $(\mathcal{B} \times \lambda)=0$.

We also observe that the Lagrangian density $\mathcal{L}_{(B, \bar{\lambda})}$ respects the following nilpotent $\left(s_{a b}^{2}=0\right)$ anti-BRST symmetry transformations $s_{(a b)}$

$$
\begin{aligned}
& s_{a b} A_{\mu}=D_{\mu} \bar{C}, \quad s_{a b} \bar{C}=-\frac{i}{2}(\bar{C} \times \bar{C}), \\
& s_{a b} C=i \bar{B}, \quad s_{a b} \bar{B}=0, \quad s_{a b} B=i(B \times \bar{C}), \\
& s_{a b} E=i(E \times \bar{C}), \quad s_{a b} \mathcal{B}=i(\mathcal{B} \times \bar{C}), \\
& s_{a b}\left(\partial_{\mu} A^{\mu}\right)=\partial_{\mu} D^{\mu} \bar{C}, \quad s_{a b} \bar{\lambda}=-i(\bar{\lambda} \times \bar{C}),
\end{aligned}
$$

because the Lagrangian density $\mathcal{L}_{(B, \bar{\lambda})}$ transforms as

$$
\begin{aligned}
s_{a b} \mathcal{L}_{(B, \bar{\lambda})} & =\partial_{\mu}\left[-\{B+(C \times \bar{C})\} \cdot \partial^{\mu} \bar{C}\right] \\
& +[B+\bar{B}+(C \times \bar{C})] \cdot D_{\mu}\left(\partial^{\mu} \bar{C}\right) \\
& -i(\mathcal{B} \times \bar{\lambda}) \cdot[\bar{B}+C \times \bar{C}],
\end{aligned}
$$

which can become a total spacetime derivative (i.e. $\left.s_{a b} \mathcal{L}_{(B, \bar{\lambda})}=\partial_{\mu}\left[B \cdot \partial^{\mu} \bar{C}\right]\right)$ provided we invoke the $\mathrm{CF}$-condition $(B+\bar{B}+C \times \bar{C}=0)$ and $(\mathcal{B} \times B)=0$. The latter restriction is also equivalent to the $\mathrm{CF}$-type restrictions $(\mathcal{B} \times \bar{\lambda})=0$ and $(B \times \bar{\lambda})=0$. Thus, we require, at least, two CF-type restrictions if we wish to have the anti-BRST invariance of $\mathcal{L}_{(B, \bar{\lambda})}$.

We end this section with the remarks on the origin of the CF-type restrictions $\mathcal{B} \times C=$ $0, \mathcal{B} \times \bar{C}=0, \mathcal{B} \times B=0$ and $\mathcal{B} \times \bar{B}=0$ which are to be invoked if we wish that the Lagrangian densities $\mathcal{L}_{(B, \bar{\lambda})}$ and $\mathcal{L}_{(\bar{B}, \lambda)}$ must respect all the four basic fermionic [i.e. (anti-) BRST and (anti-)co-BRST] symmetries of the present 2D non-Abelian theory. It is clear that the (anti-)BRST invariance of CF-condition $(B+\bar{B}+C \times \bar{C}=0)$ generates no further CF-type restriction (cf. Eqn. (13)). However, the requirement of the invariance of this CF-condition w.r.t. the (anti-)co-BRST symmetry transformations $s_{(a) d}$ leads to the new CF-type restrictions $\mathcal{B} \times \bar{C}=0$ and $\mathcal{B} \times C=0$. These restrictions are perfectly (antico-BRST invariant [i.e. $s_{(a) d}(\mathcal{B} \times C)=0, s_{(a) d}(\mathcal{B} \times \bar{C})=0$ ]. As a consequence, they have been incorporated in the Lagrangian densities through the Lagrange multipliers so that one can get rid of them. However, these restrictions are not (anti-)BRST invariant. The requirements of the (anti-)BRST invariance of these CF-type restrictions lead to the imposition of new CF-type restrictions $\mathcal{B} \times \bar{B}=0$ and $\mathcal{B} \times B=0$ which appear when we demand the (anti-)BRST invariance (i.e. $s_{a b} \mathcal{L}_{(B, \bar{\lambda})}=\partial_{\mu}\left[B \cdot \partial^{\mu} \bar{C}\right], s_{b} \mathcal{L}_{(\bar{B}, \lambda)}=\partial_{\mu}[-\bar{B}$. $\left.\partial^{\mu} C\right]$ ) of the Lagrangian densities $\mathcal{L}_{(B, \bar{\lambda})}$ and $\mathcal{L}_{(\bar{B}, \lambda)}$, respectively. We note that we do not 
need further CF-type of restrictions in our theory as far as the symmetry invariances are concerned. However, theoretically, we observe that $s_{(a) d}[\mathcal{B} \times B]=0, s_{(a) d}[\mathcal{B} \times \bar{B}]=0$ but the (anti-)BRST invariance of the restrictions $(\mathcal{B} \times B)$ and $(\mathcal{B} \times \bar{B})$ leads to new CF-type restrictions $B \times C=0$ and $\bar{B} \times \bar{C}=0$. At this stage, the series terminates because the requirements of (anti-)BRST and (anti-)co-BRST invariances produce no further new type of CF-type restrictions. The above statements can be mathematically expressed as:

$$
\begin{aligned}
& s_{d}[B+\bar{B}+(C \times \bar{C})]=0 \Longrightarrow \mathcal{B} \times \bar{C}=0, \\
& s_{a d}[B+\bar{B}+(C \times \bar{C})]=0 \Longrightarrow \mathcal{B} \times C=0, \\
& s_{b}(\mathcal{B} \times \bar{C})=0 \Longrightarrow \mathcal{B} \times \bar{B}=0, \\
& s_{a b}(\mathcal{B} \times C)=0 \Longrightarrow \mathcal{B} \times B=0, \\
& s_{b}(\mathcal{B} \times B)=0 \Longrightarrow B \times C=0, \\
& s_{a b}(\mathcal{B} \times \bar{B})=0 \Longrightarrow \bar{B} \times \bar{C}=0 .
\end{aligned}
$$

In the derivation of the above, we have used the CF-condition $(B+\bar{B}+C \times \bar{C}=0)$ as well as other CF-type restrictions that precede the derivation of any new CF-type of restriction by using the (anti-)BRST symmetry transformations. It should be noted that $s_{(a) d}[\mathcal{B} \times C, \mathcal{B} \times \bar{C}, \mathcal{B} \times \bar{B}, \mathcal{B} \times B]=0$. We point out that the requirements of $s_{(a) d}[\mathcal{B} \times C]=0$ and $s_{(a) d}[\mathcal{B} \times \bar{C}]=0$ lead to the validity of $\mathrm{CF}$-type restrictions $\mathcal{B} \times \bar{B}=0, \mathcal{B} \times B=0$. We also note and re-emphasize that all the theoretically allowed CF-type restrictions have not been used in our present discussion where we have considered only the specific kinds of infinitesimal and continuous symmetries.

\section{Symmetry operators and their algebra}

In addition to the nilpotent and absolutely anticommuting (anti-)BRST and (anti-)coBRST symmetry transformations (18), (16), (15), (14), (12) and (9), the Lagrangian densities (8) respect the following ghost-scale symmetry transformations

$$
\begin{aligned}
& C \rightarrow e^{\Omega} C, \quad \bar{C} \rightarrow e^{-\Omega} \bar{C}, \quad \lambda \rightarrow e^{\Omega} \lambda, \\
& \bar{\lambda} \rightarrow e^{-\Omega} \bar{\lambda}, \quad \Phi \rightarrow e^{0} \Phi \quad\left(\Phi=A_{\mu}, B, \bar{B}, \mathcal{B}, E\right),
\end{aligned}
$$

where $\Omega$ is a global (i.e. spacetime-independent) scale parameter and numerals in the exponents denote the ghost numbers for the corresponding fields. The infinitesimal version (with $\Omega=1$ ) of (21) are:

$$
\begin{aligned}
& s_{g} C=+C, \quad s_{g} \bar{C}=-\bar{C}, \quad s_{g} \lambda=+\lambda \\
& s_{g} \bar{\lambda}=-\bar{\lambda}, \quad s_{g} \Phi=0, \quad\left(\Phi=A_{\mu}, B, \bar{B}, \mathcal{B}, E\right) .
\end{aligned}
$$

There is a unique bosonic symmetry in our theory which is obtained from the anticommutators of the appropriate combinations of $s_{(a) b}$ and $s_{(a) d}$. We define this transformation as $\S$

\footnotetext{
${ }^{\S}$ For the sake of brevity, we have taken here only $s_{w}=\left\{s_{b}, s_{d}\right\}$ and have not considered its other definition $s_{w}=-\left\{s_{a b}, s_{a d}\right\}$. The latter definition is equivalent to it when we use the EOMs.
} 
$s_{w}=\left\{s_{b}, s_{d}\right\} \equiv-\left\{s_{a b}, s_{a d}\right\}$. The relevant fields of the theory transform, under $s_{w}$, as

$$
\begin{aligned}
& s_{w} A_{\mu}=\left[D_{\mu} \mathcal{B}+\varepsilon_{\mu \nu} \partial^{\nu} B+\varepsilon_{\mu \nu}\left(\partial^{\nu} \bar{C} \times C\right)\right] \\
& s_{w} \bar{B}=i(\bar{B} \times \mathcal{B}), s_{w}\left(\partial_{\mu} A^{\mu}\right)=\left[\partial_{\mu} D^{\mu} \mathcal{B}\right. \\
& \left.+\varepsilon^{\mu \nu}\left(\partial_{\mu} C \times \partial_{\nu} \bar{C}\right)\right], s_{w}[\bar{C}, C, B, \mathcal{B}, \bar{\lambda}]=0 \\
& s_{w} E=\left[i(E \times \mathcal{B})-D_{\mu} \partial^{\mu} B\right. \\
& \left.-\left(D_{\mu} C \times \partial^{\mu} \bar{C}\right)-\left(D_{\mu} \partial^{\mu} \bar{C} \times C\right)\right]
\end{aligned}
$$

where $s_{w}=\left\{s_{d}, s_{b}\right\}$ has been computed from the transformations (12) and (15) of the Lagrangian density $\mathcal{L}_{(B, \bar{\lambda})}$. Furthermore, the above transformations have been quoted modulo a factor of $-i$. It is straightforward to check that

$$
\begin{aligned}
s_{w} \mathcal{L}_{(B, \bar{\lambda})} & =\partial_{\mu}\left[B \cdot D^{\mu} \mathcal{B}-\mathcal{B} \cdot \partial^{\mu} B\right. \\
& \left.-\mathcal{B} \cdot\left(\partial^{\mu} \bar{C} \times C\right)+\varepsilon^{\mu \nu} B \cdot\left(\partial_{\nu} \bar{C} \times C\right)\right] .
\end{aligned}
$$

The above transformation of the Lagrangian density shows that the action integral $S=$ $\int d^{2} x \mathcal{L}_{(B, \bar{\lambda})}$ remains invariant under $s_{w}$.

It is very interesting to emphasize and observe that the above symmetry operators $s_{(a) b}, s_{(a) d}, s_{w}$ and $s_{g}$ of our present theory (corresponding to $\mathcal{L}_{(B, \bar{\lambda})}$ ) obey the following nice looking algebra:

$$
\begin{aligned}
& {\left[s_{w}, s_{r}\right]=0,(r=b, a b, d, a d, g), s_{(a) b}^{2}=0,} \\
& s_{(a) d}^{2}=0, \quad\left\{s_{d}, s_{b}\right\}=s_{w}=-\left\{s_{a b}, s_{a d}\right\}, \\
& \left\{s_{a d}, s_{d}\right\}=\left\{s_{a b}, s_{b}\right\}=\left\{s_{b}, s_{a d}\right\}=\left\{s_{d}, s_{a b}\right\}=0, \\
& {\left[s_{g}, s_{b}\right]=+s_{b}, \quad\left[s_{g}, s_{a d}\right]=+s_{a d},} \\
& {\left[s_{g}, s_{a b}\right]=-s_{a b}, \quad\left[s_{g}, s_{d}\right]=-s_{d} .}
\end{aligned}
$$

The above algebraic structure is true only when we take into account the validity of equations of motion and CF-type constraints. For instance, it can be clearly checked that $\left\{s_{b}, s_{a d}\right\} \bar{\lambda}=0$ if and only if the equation of motion $\partial_{\mu} D^{\mu} C=0$ is taken into account. Similarly, we note that $\left\{s_{b}, s_{a b}\right\} \bar{\lambda}=0$ only when we invoke the CF-type restriction $\mathcal{B} \times \bar{\lambda}=0$. A close look at the above algebra demonstrates that this algebra is reminiscent of the Hodge algebra that is obeyed by the de Rham cohomological operators ll of the differential geometry (see, e.g. [15]). They obey the following well-known algebra [15]:

$$
\begin{aligned}
& d^{2}=0, \quad \delta^{2}=0, \quad \Delta=(d+\delta)^{2}, \\
& {[\Delta, d]=0, \quad[\Delta, \delta]=0, \quad \Delta=\{d, \delta\} .}
\end{aligned}
$$

IThere is a simpler way to compute the expression for $s_{w} \mathcal{L}_{(B, \bar{\lambda})}$. This is due to the fact that $s_{w}=$ $\left\{s_{d}, s_{b}\right\}$ and the observations that $s_{b} \mathcal{L}_{(B, \bar{\lambda})}=\partial_{\mu}\left[B \cdot D^{\mu} C\right]$ and $s_{d} \mathcal{L}_{(B, \bar{\lambda})}=\partial_{\mu}\left[\mathcal{B} \cdot \partial^{\mu} \bar{C}\right]$. Thus, we have $s_{w} \mathcal{L}_{(B, \bar{\lambda})}=\left(s_{d} s_{b}+s_{b} s_{d}\right) \mathcal{L}_{(B, \bar{\lambda})}$ which leads to the derivation of $(24)$ modulo a factor of $-i$.

" On a compact manifold without a boundary, we define a set of three $(d, \delta, \Delta)$ cohomological operators which are known as the exterior derivative $d$ (with $d^{2}=0$ ), co-exterior derivative $\delta$ (with $\delta^{2}=0$ ) and the Laplacian operator $\Delta=(d+\delta)^{2} \equiv\{d, \delta\}$. These operators are popularly known as the de Rham cohomological operators of differential geometry which obey the Hodge algebra (26) where $\delta= \pm * d *$ and * is the Hodge duality operation (see. e.g. [15]). 
It is clear, from the above algebra, that the Laplacian operator behaves like a Casimir operator (but not in the Lie algebraic sense).

A precise comparison between the algebraic relations (25) and (26) demonstrates that there is a two-to-one mapping between the continuous symmetry operators and the de Rham cohomological operators of differential geometry. The explicit form of this mapping is as follows:

$$
\begin{aligned}
& \left(s_{b}, s_{a d}\right) \Rightarrow d, \quad\left(s_{d}, s_{a b}\right) \Rightarrow \delta \\
& \left\{s_{d}, s_{b}\right\}=s_{w} \equiv-\left\{s_{a d}, s_{a b}\right\} \Rightarrow \Delta .
\end{aligned}
$$

Thus, we conclude that our present 2D non-Abelian theory is a physical example of Hodge theory because the continuous transformations of the theory provide the physical realizations of de Rham cohomological operators (see, e.g. [6]).

\section{Conclusions}

One of the highlights of our present investigation is the proof of the equivalence of coupled Lagrangian densities (2) w.r.t. the (anti-)co-BRST symmetry transformations which requires new $\mathrm{CF}$-type restrictions $\mathcal{B} \times C=0$ and $\mathcal{B} \times \bar{C}=0$. These restrictions are quite different from the usual CF-condition $B+\bar{B}+(C \times \bar{C})=0$ that is required for the absolute anticommutativity of the (anti-)BRST symmetry transformations as well as for the equivalence of the Lagrangian densities (2) w.r.t. these symmetries. It is a completely new observation that the (anti-)co-BRST invariant CF-type restrictions can be incorporated into the Lagrangian densities (cf. Eqn. (8)) in such a way that both the Lagrangian densities of (8) respect the (anti-)co-BRST symmetry transformations separately and independently as is evident from (10) and (11). Thus, to have the perfect (anti-)co-BRST symmetries, we get rid of these new CF-Type restrictions in our present 2D non-Abelian 1-form gauge theory.

We have concentrated, in our present investigation, on various aspects of (anti-)coBRST symmetries of our 2D non-Abelian gauge theory (without any interaction with matter fields). We have found out that there are analogues of the CF-type condition in our 2D theory w.r.t. the (anti-)co-BRST symmetries, too (as is the case with the (anti-)BRST symmetries in the description of non-Abelian theory in any arbitrary dimension of spacetime). There are decisive differences, however, between the CF-condition associated with the (anti-)BRST symmetries and CF-type restrictions connected with the (anti-)co-BRST symmetry transformations. We point out some of the decisive differences and a few striking similarities between the CF-type restrictions from the point of view of symmetries in the next paragraphs of our present section.

First of all, the CF-condition $(B+\bar{B}+C \times \bar{C}=0)$ transforms under the (anti-)BRST symmetry transformations to itself (modulo some cross-product) as is evident from Eqn. (13). On the contrary, the CF-type restrictions $(\mathcal{B} \times C=0, \mathcal{B} \times \bar{C}=0)$ transform to zero under the (anti-)co-BRST symmetry transformations. Second, the CF-condition is somewhat hidden in the coupled Lagrangian densities in (1) and/or (2) which respect the (anti-)BRST symmetries. This condition (i.e. $B+\bar{B}+C \times \bar{C}=0$ ) can not be explicitly incorporated in the Lagrangian densities through the Lagrange multiplier while maintaining 
the perfect (anti-)BRST symmetries. In contrast, as is evident from the Lagrangian densities (8), the $\mathrm{CF}$-type restrictions $(\mathcal{B} \times C=0, \mathcal{B} \times \bar{C}=0)$ can be incorporated, through the fermionic Lagrange multipliers, in such a way that the perfect (anti-)co-BRST symmetry transformations (9) and (12) are respected by both the Lagrangian densities (8), separately and independently. Finally, the CF-condition $(B+\bar{B}+C \times \bar{C}=0)$ is responsible for the absolute anticommuting properties of the (anti-)BRST symmetry transformations. On the contrary, the CF-restrictions $(\mathcal{B} \times C=0, \mathcal{B} \times \bar{C}=0)$ are not needed for the proof of the absolute anticommutativity property of the (anti-) co-BRST symmetry transformations. These are completely novel observations.

There are a few similarities between the CF-condition associated with the (anti-) BRST symmetry transformations and the CF-type restrictions that are intimately connected with the (anti-)co-BRST symmetry transformations. The CF-condition $(B+\bar{B}+C \times \bar{C}=0)$ emerges when we apply $s_{a b}$ on $\mathcal{L}_{B}$ and $s_{b}$ on $\mathcal{L}_{\bar{B}}$ (cf. Eqn. (4)). In exactly similar fashion, the CF-type restrictions $(\mathcal{B} \times C=0, \mathcal{B} \times \bar{C}=0)$ appear when we apply $s_{a d}$ on $\mathcal{L}_{B}$ and $s_{d}$ on $\mathcal{L}_{\bar{B}}$ (cf. Eqn. (7)). It is also obvious that the Lagrangian densities $\mathcal{L}_{B}$ and $\mathcal{L}_{\bar{B}}$ (cf. Eq. (2)) turn out to be equivalent w.r.t. the (anti-)BRST as well as w.r.t. the (anti-)coBRST symmetries when we impose the CF-condition $(B+\bar{B}+C \times \bar{C}=0)$ and CF-type restrictions $(\mathcal{B} \times C=0, \mathcal{B} \times \bar{C}=0)$ together from outside. It is very interesting to note that the Lagrangian densities $\mathcal{L}_{(B, \bar{\lambda})}$ and $\mathcal{L}_{(\bar{B}, \lambda)}$ (cf. Eqn. (8)) respect three perfect symmetries but, for the (anti-)BRST invariances of these Lagrangian densities, we have to invoke the CF-condition $(B+\bar{B}+C \times \bar{C}=0)$ and CF-type restrictions $\mathcal{B} \times B=0$ and $\mathcal{B} \times \bar{B}=0$ (cf. Eqns. (17) and (19)), respectively. The latter $\mathrm{CF}$-type restrictions primarily emerge from the (anti-)BRST invarances of the CF-type restrictions $\mathcal{B} \times \bar{C}=0$ and $\mathcal{B} \times C=0$. It is an attractive idea to incorporate all the other (anti-)co-BRST invariant CF-type restrictions (e.g. $\mathcal{B} \times B=0, \mathcal{B} \times \bar{B}=0, B \times C=0, \bar{B} \times \bar{C}=0$ ) in the general form of the coupled (but equivalent) Lagrangian densities and look for the presence of the perfect (anti-)BRST and (anti-)co-BRST symmetries.

We end this section with the final remark that the absolute anticommutativity $\left(\left\{s_{b}, s_{a b}\right\}=0\right.$ and $\left.\left\{s_{d}, s_{a d}\right\}=0\right)$ properties of the (anti-)BRST and (anti-)co-BRST symmetry transformations (cf. Eqn. (25)) imply that the CF-type restrictions $\mathcal{B} \times C=0$, $\mathcal{B} \times \bar{C}=0$ should not be considered together as $s_{d}$ and $s_{a d}$ are independent of each-other. Thus, the above CF-type restrictions do not imply that $C$ and $\bar{C}$ fields are parallel (i.e. $C \times \bar{C}=0)$ in the Lie-algebraic space. As a consequence, the non-Abelian nature of the theory, with $\mathrm{CF}$-condition $(B+\bar{B}+C \times \bar{C}=0)$, is still maintained and it does not reduce to its Abelian counterpart. We can not live without $\mathrm{CF}$-condition [13] because we have proven, in our earlier works $[16,17]$, that the existence of the CF-type condition is the signature of a quantum gauge theory (described within the framework of BRST formalism) and it is deeply connected with the geometrical objects called gerbes (see, e.g. [16, 17] for details). It is gratifying that all the tower of possible CF-type restrictions for the 2D non-Abelian theory (cf. Eqn. (20)) have been derived by using the augmented version of superfield approach to BRST formalism in our recent publication [18].

\section{Acknowledgments}

N. Srinivas and S. Kumar are grateful to the financial support received from the BHU- 
fellowship under which the present investigation has been carried out.

\section{References}

[1] P. A. M. Dirac, Lectures on Quantum Mechanics, Belfer Graduate School of Science (Yeshiva University Press, New York 1964).

[2] K. Sundermeyer, Constrained Dynamics: Lecture Notes in Physics, Vol. 169 (Springer-Verlag, Berlin 1982).

[3] See, e.g., for an extensive review, R. Kumar, S. Krishna, A. Shukla and R. P. Malik, Int. J. Mod. Phys. A 29, 1450135 (2014).

[4] See, e.g., for a brief review, R. P. Malik, Int. J. Mod. Phys. A 22, 3521 (2007).

[5] R. P. Malik, Mod. Phys. Lett. A 14, 1937 (1999).

[6] R. P. Malik, J. Phys. A: Math. Gen. 34, 4167 (2001).

[7] E. Witten, Commun. Math. Phys. 117, 353 (1988).

[8] A. S. Schwarz, Lett. Math. Phys. 2, 247 (1978).

[9] N. Nakanishi and I. Ojima, Covariant Operator Formalism of Gauge Theory and Quantum Gravity (World Scientific, Singapore 1990).

[10] K. Nishijima, Czech. J. Phys. 46, 1 (1996).

[11] D. Dudal, V. E. R. Lemes, M. S. Sarandy, S. P. Sorella and M. Picariello, JHEP 0212, 008 (2002).

[12] D. Dudal, H. Verschelde, V. E. R. Lemes, M. S. Sarandy, S. P. Sorella, M. Picariello, A. Vicini and J. A. Gracey, JHEP 0306, 003 (2003).

[13] G. Curci and R. Ferrari, Phys. Lett. B 63, 51 (1976)

[14] T. Bhanja, D. Shukla and R. P. Malik, Advances in High Energy Physics 2016, 6367545 (2016).

[15] See, e.g., S. Mukhi and N. Mukunda, Introduction to Topology, Differential Geometry and Group Theory for Physicists (Wiley Eastern Pvt. Ltd., New Delhi 1990).

[16] L. Bonora and R. P. Malik, J. Phys. A 43, 375403 (2010).

[17] L. Bonora and R. P. Malik, Phys. Lett. B 655, 75 (2007).

[18] N. Srinivas and R. P. Malik, arXiv:1701.00136 [hep-th]. 